\title{
Towards a neuroscience of mind-wandering
}

\author{
1 Functional Brain Center, Wohl Institute for Advanced Imaging, Tel-Aviv Sourasky Medical Center, Tel-Aviv, Israel \\ 2 Department of Psychology, Tel-Aviv University, Tel-Aviv, Israel \\ ${ }^{3}$ Faculty of Medicine, Tel-Aviv University, Tel-Aviv, Israel \\ 4 The Emotion-Cognition Research Center, Shalvata Mental Health Center, Hod-Hasharon, Israel \\ ${ }^{5}$ Department of Neurobiology, Weizmann Institute of Science, Rehovot, Israel
}

Michal Gruberger ${ }^{1,2}$, Eti Ben-Simon ${ }^{1,3}$, Yechiel Levkovitz ${ }^{3,4}$, Abraham Zangen $^{5}$ and Talma Hendler 1,2,3*

Edited by:

Russell A. Poldrack, University of

California, USA

\section{Reviewed by:}

Georg Northoff, University of Ottawa,

Canada

Jonathan Smallwood, Max Plank

Institute for Brain and Cognition,

Germany

\section{${ }^{*}$ Correspondence:}

Talma Hendler, Functional Brain Center.

Wohl Institute for Advanced Imaging,

Tel-Aviv Sourasky Medical Center, 6

Weizmann Street, Tel-Aviv 64239,

Israel.

e-mail: talma@tasmc.health.gov.il
Mind-wandering (MW) is among the most robust and permanent expressions of human conscious awareness, classically regarded by philosophers, clinicians, and scientists as a core element of an intact sense of self. Nevertheless, the scientific exploration of MW poses unique challenges; MW is by nature a spontaneous, off task, internal mental process which is often unaware and usually difficult to control, document or replicate. Consequently, there is a lack of accepted modus operandi for exploring MW in a laboratory setup, leading to a relatively small amount of studies regarding the neural basis of $\mathrm{MW}$. In order to facilitate scientific examination of $\mathrm{MW}$ the current review categorizes recent literature into five suggested strategies. Each strategy represents a different methodology of MW research within functional neuroimaging paradigms. Particular attention is paid to resting-state brain activity and to the "default-mode" network. Since the default network is known to exert high activity levels during off-task conditions, it stands out as a compelling candidate for a neuro-biological account of mind-wandering, in itself a rest-based phenomenon. By summarizing the results within and across strategies we suggest further insights into the neural basis and adaptive value of $\mathrm{MW}$, a truly intriguing and unique human experience.

Keywords: mind-wandering, default-mode network, self, resting state, neuroimaging, fMRI, task independent thought, stimulus independent thought

"Thoughts meander like a restless wind inside a letter box they tumble blindly as they make their way across the universe" John Lennon

\section{INTRODUCTION}

Mind-wandering (MW) refers to ongoing mentation which occurs spontaneously, and largely autonomously, whenever an awake individual is not engaged in a cognitively demanding task. Alternative names to the term "MW" (Smallwood and Schooler, 2006; Mason et al., 2007) in past and recent literature include "day dreaming" (Giambra, 1979), "task-unrelated images and thought” (Giambra and Grodsky, 1989), "stimulus independent thought" (Teasdale et al., 1995), "task-unrelated thought" (Smallwood et al., 2003), "incidental self-processing" (Gilbert et al., 2005), "inner speech" (Morin, 2009), and "spontaneous thought" (Christoff et al., 2008).

Conceptualized as a core element of what William James defined as the "stream of consciousness" (James, 1892), MW, in various names and forms, has gained considerable attention in ancient and modern philosophy and in theoretical psychology. The robust, autonomous, and continual nature of this psychological process has led writers to suggest that rather than being an undesired lapse of attention to the external world (William James remarked, when he was accused of being absent-minded, that he was really just present-minded to his own thoughts; Barzun, 1983), MW must have an important adaptive value for healthy cognition (Christoff et al., 2008; Baars, 2010). Yet much like the neural basis of MW, its adaptivity and the nature of its interaction with other cognitive processes remain a scientific blind spot.
In the relatively short history of cognitive neuroscience, which has inherited much of its models, paradigms, and findings from behavioral and cognitive psychology, MW is virtually absent (Smallwood and Schooler, 2006) as a subject of research. The reluctance in the scientific arena to study MW can be accounted for by its non-behavioral characteristics when compared to more conventionally studied mental functions: MW occurs in the absence of any external cue; it is often unintended and even unaware; it takes its own course - probably driven by internally generated cues; and it is hard to trace back, replicate or report. However, a recent paradigm shift in functional neuroimaging holds a great promise for the development and establishment of MW research. The discovery of the "default-mode network" (DMN; Raichle et al., 2001) and the following realization of the significance of spontaneous resting-state neural activity (Raichle, 2009) dramatically launched a prosperous path in the scientific exploration of MW.

Default-mode network relates to a functionally meaningful neural network, which includes the medial prefrontal cortex (MPFC), the precuneus, the posterior cingulate cortex, and the inferior parietal and lateral temporal cortices (Figure 1). In comparison to other functional neural networks, DMN has unique patterns of activity (Gusnard et al., 2001; Raichle et al., 2001): both in terms of energy consumption and in terms of the blood oxygen-level dependent (BOLD) signal, activation levels in this network were shown to descend below baseline during cognitively demanding tasks. Moreover, this network shows high activation levels at rest compared to task. These activation patterns and their possible functional meaning have received considerable attention in recent years, using independent as well as combined neuroimaging techniques 


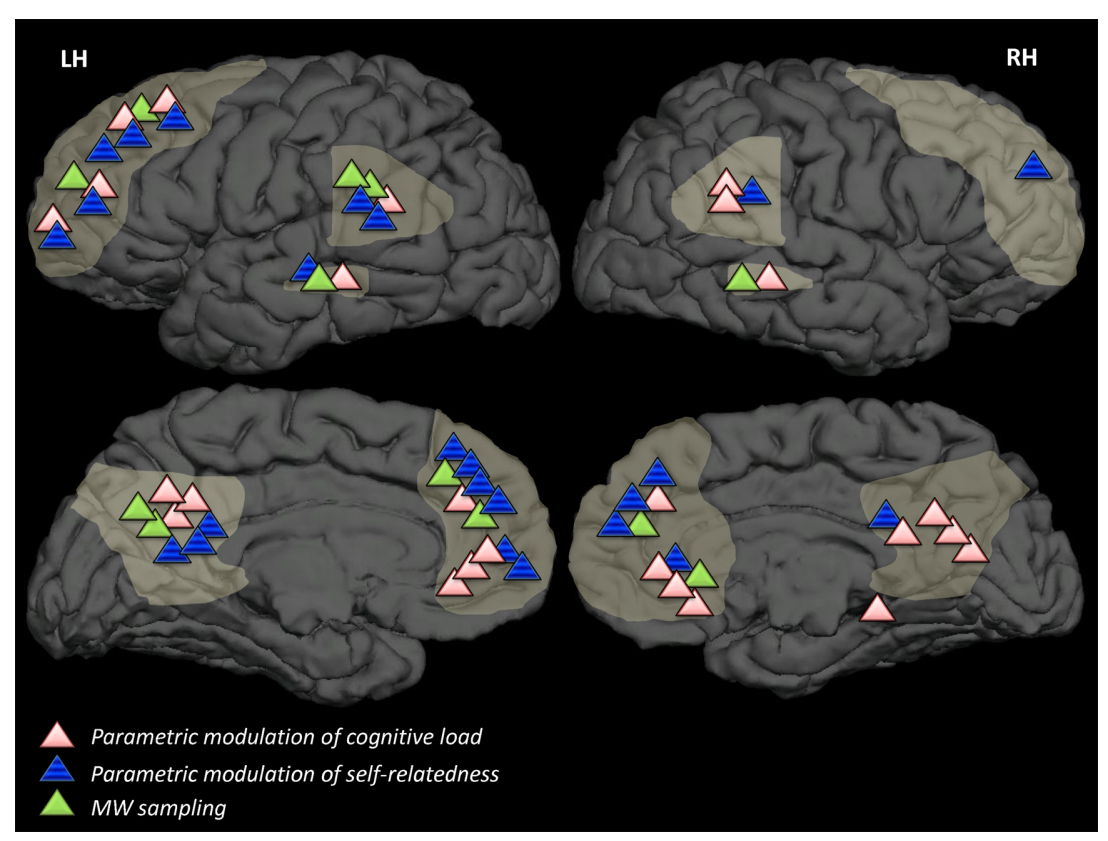

FIGURE 1 | Results of overviewed studies in relation to DMN regions.

DMN-related results of studies overviewed in this review, categorized by strategy, superimposed on a template brain. Light-grey markings denote DMN areas (in accordance with Buckner et al., 2008) dorsal and ventral medial prefrontal cortex, lateral temporal cortices, precuneus, and posterior cingulate cortex. Strategies which have not been employed in neuroimaging studies (strategy A2) or strategies which are concerned with degree of connectivity rather than degree of activation in brain areas (strategy B3) are not represented in this figure. (e.g., Ben-Simon et al., 2008). Studies with clinical populations shed additional light on the critical functionality of the DMN by demonstrating that malfunctioning of the DMN is associated with several neurological, psychiatric, and psychological pathologies (for a review see Buckner et al., 2008).

Since the first reports describing it, DMN rest-related activity had been suggested to comprise a neural correlate of MW (Gusnard et al., 2001). This proposition was based on two main features of the DMN: First of all, like MW, DMN activity occurs during rest and shows a reverse correlation with cognitive load (Mason et al., 2007). Secondly, task-related activations in the medial prefrontal and parietal areas, which comprise substantial elements of the DMN, have been shown to occur during self-related tasks (Northoff and Bermpohl, 2004; Spreng et al., 2009). This has led writers to suggest that rest-related activations in these areas might subserve MW, in itself a process of self-related mentation (Baars, 2010).

With the exceedingly growing body of information on neural activity in the wakeful, resting state, the shortage in accepted modus operandi regarding the scientific examination of MW has become a bottle neck, restraining further examination of the functionality of the DMN on the one hand and of the neural basis of MW on the other. However, several pioneering attempts have been made to study the relation between DMN activity and MW, yielding striking results. Converging the solutions to the challenge of quantifying and scientifically studying MW presented in these studies portrays an array of potential strategies to address the question of a DMN-MW association.

The current review aims to facilitate the scientific exploration of the neural correlates of MW by overviewing existing literature and defining, respectively, five methodological strategies for studying MW within a functional neuroimaging paradigm. Two of these strategies include direct measurements of MW (strategies A1 and A2), whether in real time - during rest or task performance, or retrospectively. Three additional strategies (Strategies B1, B2, and B3) rely on theoretical assumptions regarding MW and self related or cognitive functioning, as well as on the known functionality of networks emerging from connectivity analysis performed on data acquired during the resting state. Through the prism of these five strategies, we review existing literature and findings regarding MW published mainly in the recent decade. Each strategy will be presented in light of its advantages and disadvantages as well as the degree of its fitting to various paradigms and data analysis techniques in experimental neuroimaging.

\section{STRATEGIES FOR STUDYING THE NEURAL CORRELATES OF MIND-WANDERING}

The current section overviews methodologies and results from a representative sample of a decade of literature, mainly functional neuroimaging (PET or fMRI) studies, regarding the relation between DMN activation patterns and MW. The inclusion criterion for studies in this overview was that they bring forward the question of the relation between rest-related DMN activity and rest-related phenomenological experience. Importantly, studies of self-related functions were only included if they state a specific hypothesis regarding rest-related neural and psychological functioning. Table 1 lists the studies presented in this review, categorized by strategy. A visual illustration of the results obtained by these studies is presented in Figure 1.

The below differentiation between indirect and direct strategies could also be discussed in terms of determining the dependent and the independent variables within a functional neuroimaging 
Brain region/BA

\begin{tabular}{|c|c|c|c|c|c|c|}
\hline \multirow{2}{*}{ Strategy/study } & \multicolumn{3}{|c|}{ PFC } & \multicolumn{3}{|c|}{ Parietal/temporal cortex } \\
\hline & DL & DM & VM/ACC & PCC & Precuneus & TPJ \\
\hline \multicolumn{7}{|l|}{ A1. Real time MW sampling } \\
\hline Christoff et al. (2009), PNAS & & $L+R$ & $\mathrm{R}$ & & $\mathrm{L}$ & $L$ \\
\hline Stawarczyk et al. (2011), Plos One & & L & & & $\mathrm{L}$ & $\mathrm{L}$ \\
\hline \multicolumn{7}{|l|}{ B1.Parametric modulation of self-relatedness } \\
\hline Gusnard et al. (2001), PNAS & & $L+R$ & $\mathrm{~L}$ & & & \\
\hline Johnson et al. (2002), Brain & & $L+R$ & & & L & \\
\hline Goldberg et al. (2006), Neuron & $\mathrm{L}$ & L & $\mathrm{L}$ & $L+R$ & & $\mathrm{~L}$ \\
\hline Andrews-Hanna et al. (2010), Neuron & & $L+R$ & $L+R$ & & & $L+R$ \\
\hline Christoff et al. (2004), Cortex & L & & & & $L+R$ & $\mathrm{R}$ \\
\hline McKiernan et al. (2006), Neuroimage & $\mathrm{L}$ & & $L+R$ & $L+R$ & $\mathrm{R}$ & \\
\hline Mason et al. (2007), Science & $\mathrm{L}$ & $\mathrm{L}$ & $\mathrm{R}$ & & $L+R$ & $L+R$ \\
\hline B3. Data analysis of neuronal dynamics & \multicolumn{6}{|c|}{ Specific coordinates not reported } \\
\hline
\end{tabular}

This table depicts overviewed studies sorted by strategy and their DMN-related results. Areas of DMN were chosen with accordance to Buckner et al. (2008). Numbers represent Brodmann areas. PFC, prefrontal cortex; DL, dorso lateral; DM, dorso medial; VM, ventro medial; P/ACC, posterior/anterior cingulate cortex; TPJ, temporoparietal junction.

setup: in the case of the indirect strategies, cognitive load or selfrelatedness are being experimentally manipulated (i.e., independent variable) and are expected to cause a change in the measured neural signal of the DMN (i.e., dependent variable); in the case of the direct strategies, the degree of DMN activation is manipulated (i.e., independent variable) by altering rest and task while scanning, and the consequent change in the degree of MW is being assessed following scanning (i.e., dependent variable). Table A2 in Appendix summarizes typical dependent and independent variables according to each strategy.

\section{DIRECT STRATEGIES FOR MEASURING MIND-WANDERING}

Strategies for directly quantifying the degree of MW represent a straightforward attempt to overcome its non-explicit nature, and essentially make conventional experimental methods applicable for studying it. For example, one can use the obtained degree of reported MW to categorize sessions or subjects into groups before analyzing, or to correlate it with the degree of activation in selected brain regions of interest or in the whole brain. The greatest challenge, however, is that in contrast to most behavioral measurements, the actual tracking of MW, or even its mere verbalizing in real time, tampers with its very occurrence (Filler and Giambra, 1973): an individual busy with reporting her own MW is less free to engage in spontaneous MW comparing to when left to rest quietly. This can probably account for the relatively few studies which have attempted to directly quantify MW in the history of cognitive neuroscience, and possibly, for the even fewer methods developed to do so. Several quantifications tech- niques have nonetheless, been employed, some attempting at realtime assessment of the degree of MW while others focusing on post hoc questioning of subjects.

\section{Strategy A1: real-time MW sampling}

Mind-wandering can occur with or without awareness of its occurrence ("meta-awareness"; Christoff et al., 2009). Nevertheless, one can normally report if a thought was occurring in their mind or not, if interrupted and asked to do so at a given time point. This is the rational underlying the MW sampling (also known as "thought sampling" or "thought probing") technique (McKiernan et al., 2006; Mason et al., 2007; Christoff et al., 2009; Stawarczyk et al., 2011). Several approaches have been introduced for MW sampling in the neuroimaging set up, but a typical one uses a probing tone in even or uneven intervals, during either a rest or a task scan; subjects are instructed to indicate whether they were experiencing a spontaneous thought (i.e., unrelated to task performance) at the time the tone was presented (or, in a similar version, since the previous probe; Giambra, 1995). In block-design neuroimaging studies, each scan session is then scored according to the rate of "yes" answers given in it out of the overall number of tones presented in the session. The degree of MW occurrence is found to correlate with degree of neural activity in the DMN, as illustrated in Figure 1. In ERP studies, EEG recordings adjacent to the pressings are analyzed separately for "on-task" vs. "off-task" reports. Using this method, Kam et al. (2011) demonstrated that the P1 component to a visual or auditory probe was reduced during off task, implying a reduction in sensory level processing during MW. 
An interesting body of research based on this strategy examines the link between MW (referred to as "task-unrelated thought") and errors during task performance (Smallwood et al., 2003, 2008). These studies demonstrate that when MW intrudes during task performance, and attentional lapses occur, task performance is impaired. Based on this line of research it may be suggested that MW competes with task performance on a limited capacity of attentional resources, in effect representing a state of "perceptual decoupling" (Smallwood et al., 2011a). This corresponds well with the idea discussed later on in this paper on the reverse correlation between MW and executive networks in the brain.

In yet another, less common, version of MW sampling, subjects are requested to press a button each time a thought comes into mind (Giambra, 1989). Using this type of report, Braboszcz and Delorme (2010) asked subjects to press a button as soon as they realized their mind was wandering during a task of counting breaths. These presses were later used as an ERP analysis, showing reduced P200 responses to auditory stimuli, and reduced ability to identify the oddball auditory stimuli (smaller N100 during MW). In addition, frequency analysis showed that MW was associated with higher delta and theta power and lower alpha and beta power compared to task performance. Despite the suitability of this method for ERP studies, as reported by the authors themselves, this version seems to be less favorable and can hardly be found in neuroimaging studies, probably because it imposes greater meta-awareness and concentration from subjects and thus interferes with the natural occurrence of MW.

The strategy of MW sampling presents a clear advantage of being a real time, direct and quantified measurement of MW occurrence. One should bear in mind, though, that to the best of our knowledge it has never been systemically tested for validity and reliability, and thus it is mainly justified by its straight-forwardness and intuitiveness.

\section{Strategy A2: retrospective evaluation of MW}

Mind-wandering requires peace of mind; disturbances tend to interrupt its natural flow. In other words, an informative report regarding $\mathrm{MW}$ at a given time period, without interfering with its occurrence, may better be collected retrospectively, after a session has ended (notably, even then, the contents of MW is not always straightforwardly accessible to memory). Surprisingly, though, designated structured psychological questionnaires for explicitly assessing MW in healthy individuals are scarce. The very few examples which can be found in the literature (Giambra, 1979; Klinger and Cox, 1987; Matthews et al., 1999) did not seem to survive the transition from psychological behavioral research to neuroscience. Consequently, and unfortunately, there is no accumulated body of literature regarding the neural basis of MW, and virtually no experience in the field obtained by retrospective questioning of MW using validated experimental instruments designated for this matter.

One inspiring study which could be considered an example for this approach is a PET study by D'Argembeau et al. (2005). In this study subjects had to rate the total amount of thoughts experienced, whatever their content, using an in-house developed questionnaire immediately following scanning (a similar approach is found earlier in Mcguire et al. 1996). An alternative to using in-house developed questionnaires is to use established questionnaires of experiences which according to theoretical and clinical literature are related to MW. In such a study (Gruberger et al., 2008), questionnaires for measuring dependent self awareness and degree of dissociation were applied to assess the degree of interference in MW during rest. The underlying hypothesis was that artificial interference with the normal process of MW will manifest itself as disruption in self awareness and as a sense of dissociation, which indeed was corroborated by the results. A third noteworthy example is the Resting State Questionnaire (ReSQ) published recently by Delamillieure et al. (2010) explicitly for usage in a functional neuroimaging setup. The ReSQ consists of 62 items organized by five main types of mental activity: visual mental imagery, inner language, somatosensory awareness, inner musical experience, and mental manipulation of numbers. Using a $0-100 \%$ scale, the participant retrospectively and quantitatively rates the proportion of time spent in each mental activity during the resting-state fMRI acquisition. Whether this tool will or will not eventually gain the confidence of the research community, its great importance lies in that it represents a pioneering effort to encompass the richness and individual nature of MW into a standardized questionnaire.

\section{INDIRECT STRATEGIES FOR MEASURING MIND-WANDERING}

Indirect strategies - strategies in which MW is not directly measured - are typically based on the conceptualization of MW as self-related and as more prevalent during rest than during tasks of high cognitive demand. The hypothesis could be framed as follows: if DMN neural activity during rest is the neural basis of MW, then DMN activations during rest and during a given task should be more similar when the task shares common characteristics to MW, i.e., is characterized by low cognitive load and high self relevance.

The advantage of the indirect strategies is straightforward: they avoid measuring MW directly, thus overcoming its non-quantifiable nature and the lack of validated behavioral MW measures. Instead, they use accepted task-related behavioral measures (mostly validated or previously published) and modulate their self-relatedness or their degree of cognitive load.

\section{Strategy B1: parametric modulation of self-relatedness}

James's "spiritual self" (James, 1892), Gallagher's "narrative self" (Gallagher, 2000), Dennett's “non-minimal self” (Dennett, 1991), and Damasio's “autobiographical self” (Damasio, 1998), are just a few examples of how MW is often present within theoretical models of the self. It is typically represented as a module of its own, distinct both from "lower," more basic, senses of consciousness as well as from "higher" self-related executive functions. Contemporary neuro-scientists also tend to agree that the "stream of consciousness" is inseparable from the ongoing, constant, sense of self (Damasio, 1998; Gusnard, 2005; Beer, 2007). According to this notion, MW, whether its content is directly related to the thinker or not, is a self-related, self-generated, self-sustaining function (Baars, 2010); it serves as an integral part of self awareness, a pre-requisite for healthy psychological functioning.

The conceptualization of MW as a private case of self-related functioning produces a hypothesis for an overlap between the neural basis of self-related tasks and the neural basis of MW. This hypothesis has been translated in some studies into a rational for comparing neural activations during self-related tasks to neural activations at rest, when MW is assumed to occur most. 
Though not the first to suggest a relation between rest-related neural activity and MW, the first paper to specifically associate MW with DMN activity was published by Gusnard et al. (2001), as part of a series of publications (Raichle et al., 2001) introducing the concept of the DMN. In this fMRI study, neural activations during rest were compared both to a subjective, emotional judgment task ("internally cued condition") and to a neutral judgment task ("externally cued condition"). In accordance with the above prediction, neural activations in DMN-related PFC areas were found to be more similar to the activations at rest during the internally cued condition than during the externally cued condition (see Table 1 for summarized results). Paradigms similar in contrasting a self-related task with a similar non-self-related task can be found in additional fMRI studies (Johnson et al., 2002; Goldberg et al., 2006; Schneider et al., 2008; Andrews-Hanna et al., 2010), and in the PET study described earlier (D'Argembeau et al., 2005). Results in all of these studies indicate greater activations (or lesser deactivations) in brain areas associated with the DMN, mostly MPFC areas, during self-related tasks than during non-self-related tasks, when compared to rest. These elevated activations were shown to last beyond the duration of the stimuli and into the rest period following stimulation (Schneider et al., 2008). The majority of these papers (except for Johnson et al., 2002) demonstrate that DMN activations during self-related conditions were more similar to DMN rest-related activity patterns, and suggest that this result might imply a possible functional role of rest-related DMN activations in spontaneous self-related mental activity.

As shown in separate studies as well as in convergence, this is a useful strategy for investigating the functional role of areas within the DMN while staying within the boundaries of accepted neuroimaging paradigms. One drawback of this strategy is the potential of over stretching the concept of self, which may cause confounding the self-relatedness of a task with other characteristics like its emotional valence (e.g., Gusnard et al., 2001). Therefore, one should pay special attention that the parameter modulated between study conditions is indeed as specific to self-relatedness as possible.

\section{Strategy B2: parametric modulation of cognitive load}

The distinction of ongoing spontaneous mentation from other, task related, mental functions dates back to James (1892), and has been recognized almost solely by theoretical psychology and philosophy over the years (Gallagher, 2000). However, this very classification of MW as the mental function characterizing the un-engagement of attentional resources directly magnifies its potential to be scientifically explored.

The strategy of parametric modulation of cognitive load has been used in the context of studying the functionality of restrelated DMN activity. In this strategy, the contrast of interest when analyzing imaging data is not the commonly used task minus rest contrast, but rather the contrast of rest minus task. Researchers try to demonstrate that the lower the cognitive load in a given task condition, the higher the activations in DMN areas during this task, leading to a smaller difference between DMN activations during the task compared to rest. Indeed, this was found to be the case in fMRI studies such as McKiernan et al. (2006), Christoff et al. (2004), and Mason et al. (2007), and in Wicker et al.'s (2003) meta-analysis of PET studies. In the case of McKiernan et al. (2006) and Mason et al. (2007), behavioral measures (described in strategy A1) were added to the study to further establish a more direct association between high DMN activations during low cognitive demand and MW.

This strategy yields results which correspond well with theoretical accounts of MW as well as with the lay intuition that MW is the "default" mental state when the mind is free to engage in it. In addition to its intuitiveness, and thus its simplicity, the advantage of this strategy is in its robustness: it was found to be replicated across virtually any behavioral task tested (Shulman et al., 1997; Mazoyer et al., 2001; Wicker et al., 2003), which makes it accessible and easy to implement. It should be taken into account, however, that executive functioning and MW are probably not as anti-correlated as these studies may depict. MW may involve executive processes like memory, planning, computing, etc., as is reflected by findings of executive networks co-activated with DMN during MW (Christoff et al., 2009). Thus, rather than assuming mutual exclusiveness, the degree and direction of the association between neural activity of the DMN and of executive networks during MW should be studied in greater experimental resolution.

\section{Strategy B3: paradigm-free analysis of neuronal dynamics}

Brain activity is combined of activations of neurons which comprise anatomical and functional networks. Recent advances in functional and computational neuroimaging have provided new tools for examining functional interactions between time series of signals obtained from different brain regions, catalyzing the examination of functional connectivity in the resting brain. This type of analysis does not require a behavioral paradigm ("paradigm-free") and indeed is often implemented on data acquired solely when subjects lie resting in the imaging device (the validity of these signals is discussed in Box 1). In fMRI, analysis methods of the restingstate signal can typically be placed into hypothesis dependent and hypothesis free methods (Van Den Heuvel and Hulshoff Pol, 2010), both resulting in connectivity maps - whether correlational or anticorrelational (Uddin et al., 2009). These maps demonstrate anatomical networks which, interestingly, greatly overlap with known functional neural networks. The DMN is one of those emerging networks and thus its relation to MW can be further characterized in terms of functional connectivity.

Two studies are brought here to exemplify the usage of a paradigm-free strategy in further characterizing the relation between MW and DMN spatio-temporal dynamics. Horovitz et al. (2008) utilized this strategy to determine whether DMN activity can be de-coupled from conscious awareness. In this study, the level of functional connectivity within the DMN persisted both during the resting state and during light sleep. The authors conclude that DMN connectivity "does not require or reflect the level of consciousness that is typical for wakefulness" (p. 679), which seems to undermine the idea of a functional involvement of DMN activity in MW. Nevertheless, two alternative explanations are offered by the authors: the first is that these results only decouple wakeful awareness from the degree of connectivity within the DMN, but not from the amplitude of its activity; the other is that light sleep is sometimes characterized by the existence of dream-like reverie activity (a mental activity similar to MW) which like MW may also be a functional product of DMN activity. 


\section{BOX 1 | Validation of spontaneous BOLD fluctuations acquired during rest.}

The neuronal basis of spontaneous resting-state fMRI signals was initially regarded by skeptics as problematic, potentially representing merely unknown parameters of noise as well as known physiological ones. However recent observations increasingly support and validate the neuronal basis of resting-state fMRI signals (Adapted from Van Den Heuvel and Hulshoff Pol, 2010):

- The first and probably most compelling evidence for the resting-state signal is that most resting-state patterns tend to occur between brain regions overlapping in known functional and neuroanatomical regions (Salvador et al., 2005; Damoiseaux et al., 2006; Van Den Heuvel et al., 2008).

- The second observation relates to the frequency of restrelated signals revealing that the observed spontaneous BOLD signals are mainly dominated by lower frequen- cies $(<0.1 \mathrm{~Hz})$ with only a minimal contribution of higher frequency cardiac and respiratory oscillations $(>0.3 \mathrm{~Hz})$ (Cordes et al., 2000, 2001).

- Lastly, an (indirect) association exists between the frequency profiles of slow spontaneous resting-state fMRI and electrophysiological recordings of neuronal firing (Nir et al., 2008) and between spontaneous BOLD fluctuations and simultaneous measured fluctuations in neuronal spiking (Shmuel et al., 2002; Shmuel and Leopold, 2008).

Altogether these findings advocate toward the validity of the neural signal acquired during the resting state and the legitimacy of its scientific exploration.
Another study by the same group (Horovitz et al., 2009) demonstrated altered correlations between DMN network components during different states of consciousness, most notably a reduced involvement of the MPFC during sleep. The authors suggest that among the DMN components, the frontal cortex may play an important role in the sustenance of conscious awareness.

In favor of this strategy, it can be claimed that as some indication exists for the effect of previous task performance on neural activity at subsequent rest (Northoff et al., 2010), a paradigm-free study design which consists of rest alone will produce results which are more unbiased. In any case, studies of this strategy call attention to the fact that beyond relative degree of neural activity, more holistic parameters of neural dynamics need to be explored to truly characterize the DMN-MW relation, such as temporal and spatial patterns of DMN activity.

\section{DISCUSSION AND FUTURE DIRECTIONS}

In this review we portray the evolvement of the neuroscience of $\mathrm{MW}$, in hope to lay the grounds for additional research to come. Undoubtfully, studies like the ones overviewed here serve to narrow the gap between theoretical understanding of MW and its scientific exploration. Nevertheless, MW is still by large a mystery, and much work remains to complete the puzzle. In Box 2 we put forward several ideas which stem from existing findings in hope of contributing to future research.

Mind-wandering can be studied under different contexts involving a wide array of experimental questions. Accordingly, as we tried to exemplify in this review, there is no absolute optimal way to study it, but rather it is important to make an informed, educated choice when studying it within a neuroimaging paradigm. For instance, MW sampling provides valuable information about inter-subject and intra-subject differences in the degree of MW, while sacrificing the integrity of its natural, untouched flow; In contrast parametric modulation of cognitive load does not interfere with the natural course of MW and also enables statistical analysis of inter-group variance, with the compromise of MW being only implied, and not directly measured. Figure 2 depicts a flow chart of relevant considerations in making the most advantageous choice for a given experimental setup.
In addition to portraying modes of operation for the scientific examination of MW, overviewing the neuroscience of MW so far provides a few insights into this neural and mental process.

\section{FUNCTIONALITY OF MIND-WANDERING}

The robustness of the experience of MW across ages, cultures, and individuals (Singer and McCraven, 1961), suggests it holds a vital role in human psychology. As to the specific role of MW, we suggest several ideas based on current literature which may inspire future research.

\section{MW serves "self" functions}

As detailed in the context of strategy B1, there are theoretical (Gallagher, 2000), neuroanatomical (Gusnard, 2005; Northoff et al., 2006), and intuitive grounds to claim that MW is a selfrelated cognitive function, which serves to create and maintain an integrated, meaningful sense of self out of various aspects of self-related information and cognition. Northoff et al. (2006), for instance, conceptualizes MW as a "psychological baseline," a form of continuous self-referential processing which is evident during non-task conditions and which ultimately forms our subjective experience of a "continuous stream of subjective experience" or "phenomenal time" where past, present, and future are no longer divided but integrated.

\section{MW enables the projection of a "self" to past and future events}

The idea that MW serves processes of future planning and simulation is based on theory and common experience, and is strongly supported by the fact that the DMN includes areas such as the posterior cingulate cortex, the precuneus, and the hippocampus, which are known to take part in such mental processes (Buckner et al., 2008). Behaviorally, it has been shown that the contents of MW will tend toward prospecting or retrospecting according to the self relevance of a given context (Smallwood et al., 2009), suggesting that MW serves to integrate past and present experiences for the purpose of future planning. Moreover, Smallwood et al. (2011b) suggest that self reflection associated with future-oriented thinking is an integral part of the autobiographical memory system. Interestingly, temporal locus of MW has even been shown 


\section{BOX 2 | Mind-wandering: questions for future research.}

Understanding MW using brain imaging techniques holds a promise for this field of research. Listed here are a few lines of thought that could constitute an initial framework for future MW studies:

A. Temporal patterns of $M W$ : What are the spatio-temporal dynamics which correspond to MW in the human brain? How are they represented in terms of brain connectivity?

B. Control of MW: what is MW's locus of control in the brain? Do internal and external abruptions of MW result in similar neural outcome? Interfering with MW occurrence by different type of tasks (e.g., tasks which require external vs. internal attention) could offer preliminary answers.

C. MW and consciousness: What is the nature of the relationship between consciousness and MW? Is MW simply an expression of conscious experience much like an actor on a stage or is it a substantial part of consciousness giving rise to the stage itself? If MW is indeed a substantial part of conscious experience one would expect similar neural correlates of both phenomena.

D. MW and pathologies: Which functions does MW serve and how are they disrupted when MW does not occur? Both the very mechanism and the contents of MW are of great interest to clinical psychology and psychiatry. Psychiatric and neuronal pathologies associated with MW malfunctioning may shed light on understanding the role of MW in healthy psychological functioning.

E. The contents of MW: In this review we put little emphasis on the ever changing contents of MW. This is not to say that they are of no importance, only that the studies described here were interested in the common mechanism underlying this changing flow of contents. Future research might very well attempt to segregate neural patterns during MW which are responsible for the experience of different contents or even different time directions (e.g., future or past) as explored by Smallwood et al. (2009).

To begin answering such questions, the scientific community must agree upon theoretical definitions as well as normalized, standardized behavioral measures of MW. In the functional neuroimaging field one also needs advanced validated computational methods for studying the temporal dynamics of neural activations in long sequences such as common in rest.

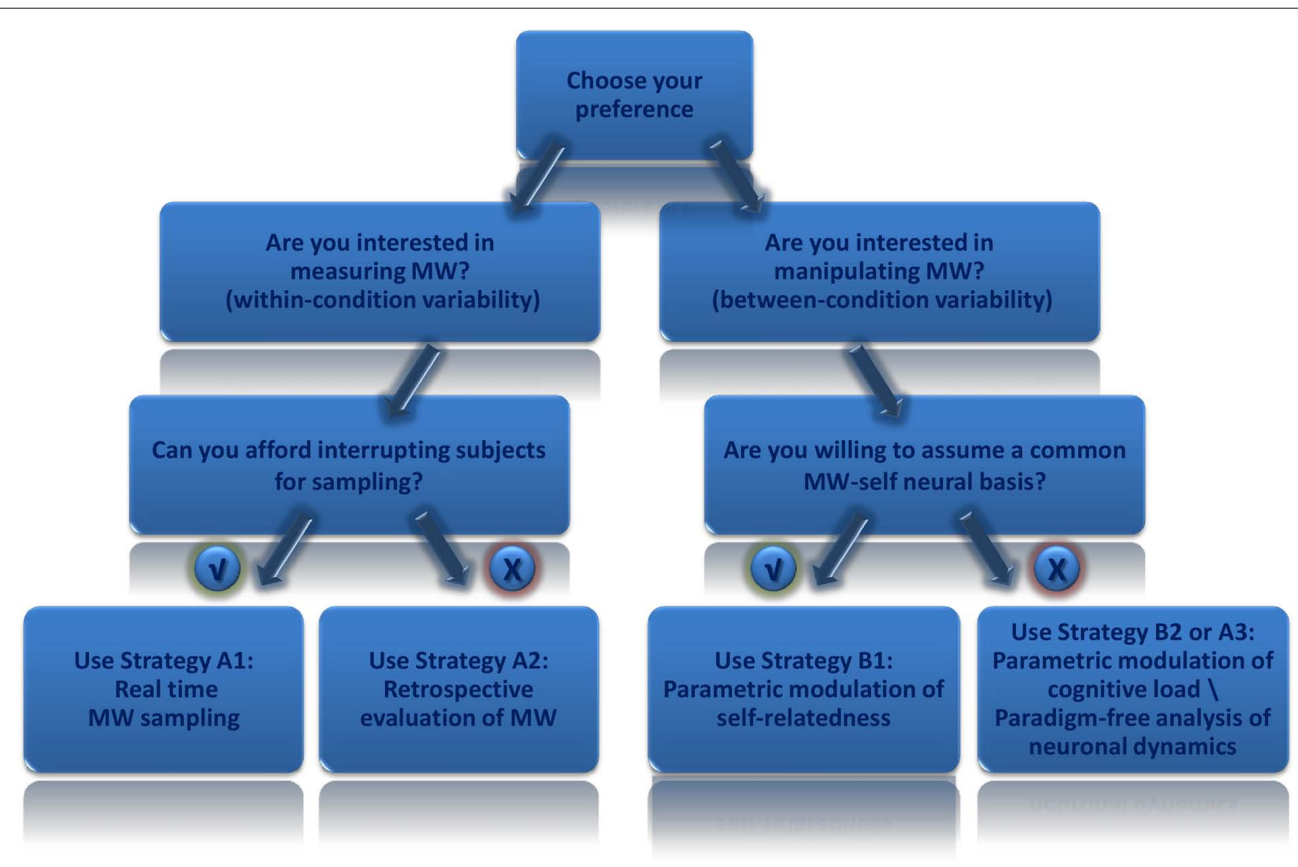

FIGURE 2 | Flowchart of goodness of fit of different strategies according to study aims. A flowchart which may assist researchers wishing to explore mind-wandering using functional neuroimaging paradigms. According to study aims one should decide on the appropriate strategy taking into consideration advantages and disadvantages of each strategy as discussed in the text.

to be related to the direction of apparent physical movement through space (forward/backward), implying a functional link between MW temporality and sensory spatio-temporal input (Miles et al., 2010).
Altogether, this idea corresponds well with Tulving's idea of "autonoetic consciousness," which is claimed to be selective to the human kind and which enables mentally traveling into the past and the future (Tulving, 2005). 


\section{MW serves as a learning and consolidation mechanism by augmenting the associative abilities of the brain}

According to this proposition, spontaneous mental processing during wakefulness resembles in its function, in its effects and, to a certain extent, in its neural basis, the off-line processing that occurs during sleep. This relatively recent idea is presented by contemporary writers (Christoff et al., 2008; Baars, 2010) and already takes into account what is known about DMN activation patterns. According to this notion, it could be suggested that task performance would improve following MW in a similar way when following sleep (Stickgold et al., 2001).

\section{MIND-WANDERING-EXECUTIVE FUNCTIONING RELATION: AN INTEGRATIVE APPROACH}

Converging results from studies like the ones overviewed here provide verification for a strong negative association between MW and executive functioning. This association, mentioned earlier to be part of the rational for strategy B2 (Parametric modulation of cognitive load), is supported by behavioral as well as neuroscientific evidence (e.g., DMN activity). In light of the infancy of MW research, this in itself is a highly instrumental insight.

Nevertheless, recent lines of evidence suggest that this association is not exclusive. The first is found in the activation of executive prefrontal and parietal brain areas, in addition to DMN areas, as contributing to MW (Christoff et al., 2009). The second is found in the gradual increase of DMN activity found in strategies B1 and B2 as cognitive load decreases and self-relatedness increases, which suggests that some DMN activity did occur even in lower self relevance or higher cognitive load conditions. The third line of evidence is brought by studies which show involvement of DMN areas during online task performance (Assaf et al., 2009).

Though assuming a dichotomy between MW and "executive" neural networks proved useful for the beginning of MW research, a more mature approach might suggest studying the interplay between MW and executive functions and their underlying neural mechanisms (Smallwood and Schooler, 2006). In consistence with this line of thought, Spreng et al. (2010) suggest that a third anatomically interposed "frontoparietal control network" mediates planning across domains, flexibly coupling with either the default or dorsal attention network in support of internally vs. externally focused cognition, respectively.

Rather than eliminating them, MW probably serves various cognitive functions such as prospective planning, self monitoring, etc. (Baars, 2010). A better understanding of the interplay between MW and executive functioning can be achieved by further implementation of the five strategies defined here, in turn contributing altogether to the understanding of the adaptive value of MW with respect to human cognition and affect.

\section{REFERENCES}

Andrews-Hanna, J. R., Reidler, J. S., Sepulcre, J., Poulin, R., and Buckner, R. L. (2010). Functional-anatomic fractionation of the brain's default network. Neuron 65, 550.

Assaf,M., Kahn,I., Pearlson, G.D., Johnson, M.R., Yeshurun, Y., Calhoun, V.D., and

\section{MIND-WANDERING: THE NEURAL BASIS OF ITS INTEGRATION AND SEGREGATION}

Portraying the results of the overviewed studies suggests that MW involves activities in distributed brain areas (see Table $\mathbf{1}$ and Table A1 in Appendix). These findings of different activations might underlie specific aspects of the MW process and in turn may serve to deconstruct MW, both theoretically and operationally, into elements according to its content or to the additional mental functions which are involved in it (e.g., emotion, autobiographical memory, mental time traveling, etc.). Examining the different DMN activations according to strategy, as illustrated in Figure 1, implies that some sub-areas within the DMN are common to $\mathrm{MW}$ in any context while others are more typically unique to a specific strategy. For example, on an impressionist level only, it could be suggested that across strategies lateral correlates of MW are found more dominantly in the left than in the right hemisphere and can be commonly regarded as part of the network associated with high-level semantic processing. However other correlates of MW do differ between strategies, with the ventral MPFC and precuneus more sensitive to modulation of cognitive load, and dorso-medial MPFC areas more sensitive to self-relatedness.

It is of no doubt that such impressions require a comprehensive quantitative meta-analysis which is beyond the scope of this review. Nevertheless, such a neuro-functional differentiation implies that each strategy might reveal, in addition to the network underlying MW, the neural basis of a specific aspect within the large construct of MW. A functionally based deconstruction of the DMN has already been suggested (Spreng et al., 2009; Andrews-Hanna et al., 2010; Stawarczyk et al., 2011) and could prove fruitful for further scientific examination of MW; Similar MW studies utilizing such refined and specific definitions may shed additional light on differential neural processes which underlie diverse aspects of MW.

\section{CONCLUDING REMARKS}

Mind-wandering is a universal phenomenon which accompanies much of our daily lives from childhood to adulthood. Its exploration has a vast potential in leading us to a better and more profound understanding of our ongoing mental selves, and in fact, of the basic properties of conscious experience.

The study of MW is at an exciting position of forming into a field of research of its own. Its relevance to a wide array of disciplines, from neuroscience to philosophy to the clinical world ensures that it will draw a growing number of researchers in the near future. We hope that this review serves to set the milestones for a better scientific understanding of this remarkable, unique human quality.

Barzun, J. (1983). A Stroll with William James. New York, NY: HarperCollins. Beer, J. S. (2007). The default self: feeling good or being right? Trends Cogn. Sci. (Regul. Ed.) 11, 187-189.

Ben-Simon, E., Podlipsky, I., Arieli, A., Zhdanov, A., and Hendler, T. (2008). Never resting brain: simultaneous representation of two alpha related processes in humans. PLoS ONE 3, e3984. doi: 10.1371/journal. pone.0003984

Braboszcz, C., and Delorme, A. (2010). Lost in thoughts: neural markers of low alertness during mind wandering. Neuroimage 54, 3040-3047. 
Buckner, R., Andrews-Hanna, J., and Schacter, D. (2008). The brain's default network. Ann. N. Y.Acad. Sci.1124, 1-38.

Christoff, K., Gordon, A., Smith, R., and Vancouver, B. C. (2008). "The role of spontaneous thought in human cognition," in Neuroscience of Decision Making, ed. O. A. M. Vartanian (Philadelphia: Psychology Press).

Christoff, K., Gordon, A. M., Smallwood, J., Smith, R., and Schooler, J. W. (2009). Experience sampling during fMRI reveals default network and executive system contributions to mind wandering. Proc. Natl. Acad. Sci. U.S.A. 106, 8719-8724.

Christoff, K., Ream, J. M., and Gabrieli, J. D. (2004). Neural basis of spontaneous thought processes. Cortex 40 , 623-630.

Cordes, D., Haughton, V. M., Arfanakis, K., Carew, J. D., Turski, P. A., Moritz, C. H., Quigley, M. A., and Meyerand, M. E. (2001). Frequencies contributing to functional connectivity in the cerebral cortex in "resting-state" data. AJNR Am. J. Neuroradiol. 22, 1326.

Cordes, D., Haughton, V. M., Arfanakis, K., Wendt, G. J., Turski, P. A., Moritz, C. H., Quigley, M. A., and Meyerand, M. E. (2000). Mapping functionally related regions of brain with functional connectivity MR imaging. AJNR Am. J. Neuroradiol. 21, 1636.

D’Argembeau, A., Collette, F., Van Der Linden, M., Laureys, S., Del Fiore, G., Degueldre, C., Luxen, A., and Salmon, E. (2005). Self-referential reflective activity and its relationship with rest: a PET study. Neuroimage 25, 616-624.

Damasio, A. R. (1998). Investigating the biology of consciousness. Philos. Trans. R. Soc. Lond. B Biol. Sci. 1879-1882.

Damoiseaux, J.S., Rombouts, S., Barkhof, F., Scheltens, P., Stam, C. J., Smith, S. M., and Beckmann, C. F. (2006). Consistent resting-state networks across healthy subjects. Proc. Natl. Acad. Sci. U.S.A. 103, 13848.

Delamillieure, P., Doucet, G., Mazoyer, B., Turbelin, M.-R., Delcroix, N., Mellet, E., Zago, L., Crivello, F., Petit, L., Tzourio-Mazoyer, N., and Joliot, M. (2010). The resting state questionnaire: An introspective questionnaire for evaluation of inner experience during the conscious resting state. Brain Res. Bull. 81, 565-573.

Dennett, D. C. (1991). Consciousness Explained. Boston: Little Brown \& Co.

Filler, M. S., and Giambra, L. M. (1973). Daydreaming as a function of cueing and task difficulty. Percept. Mot. Skills $37,503$.

Gallagher, S. (2000). Philosophical conceptions of the self: implications for cognitive science. Trends Cogn. Sci. (Regul. Ed.) 4, 14-21.
Giambra, L. M. (1979). Sex differences in daydreaming and related mental activity from the late teens to the early nineties. Int. J. Aging Hum. Dev. 10, 1-34.

Giambra, L. M. (1989). Task-unrelatedthought frequency as a function of age: a laboratory study. Psychol. Aging 4, 136-143.

Giambra, L. M. (1995). A laboratory method for investigating influences on switching attention to task-unrelated imagery and thought. Conscious. Cogn. 4, 1-21.

Giambra, L. M., and Grodsky, A. (1989). Task-unrelated images and thoughts while reading. Imagery Curr. Perspect. 26-31.

Gilbert, S. J., Frith, C. D., and Burgess, P. W. (2005). Involvement of rostral prefrontal cortex in selection between stimulus-oriented and stimulus-independent thought. Eur. J. Neurosci. 21, 1423-1431.

Goldberg, I. I., Harel, M., and Malach, R. (2006). When the brain loses its self: prefrontal inactivation during sensorimotor processing. Neuron 50, 329-339.

Gruberger, M., Hendelr, T., Harel, E. V., Harari, H., Levkovitz, Y., and Zangen, A. (2008). I think therefore I am: alterations in the sense of self by stimulation of the prefrontal cortex. NeuroImage, 41, S41-S180.

Gusnard, D. A. (2005). Being a self: considerations from functional imaging. Conscious. Cogn. 14, 679-697.

Gusnard, D. A.,Akbudak, E., Shulman, G. L., and Raichle, M. E. (2001). Medial prefrontal cortex and self-referential mental activity: relation to a default mode of brain function. Proc. Natl. Acad. Sci. U.S.A. 98, 4259-4264.

Horovitz, S. G., Braun, A. R., Carr, W. S., Picchioni, D., Balkin, T. J., Fukunaga, M., and Duyn, J.H. (2009). Decoupling of the brain's default mode network during deep sleep. Proc. Natl. Acad. Sci. U.S.A. 106, 11376.

Horovitz, S. G., Fukunaga, M., De Zwart, J. A., Van Gelderen, P., Fulton, S. C., Balkin, T. J., and Duyn, J. H. (2008). Low frequency BOLD fluctuations during resting wakefulness and light sleep: a simultaneous EEG fMRI study. Hum. Brain Mapp. 29, 671-682.

James, W. (1892). Psychology. Cleveland: World.

Johnson, S. C., Baxter, L. C., Wilder, L. S., Pipe, J. G., Heiserman, J. E., and Prigatano, G. P. (2002). Neural correlates of self reflection. Brain 125 , 1808-1814.

Kam, J.W.Y., Dao, E., Farley, J., Fitzpatrick, K., Smallwood, J., Schooler, J. W., and Handy, T. C. (2011). Slow fluctuations in attentional control of sensory cortex. J. Cogn. Neurosci. 23, 460-470.
Klinger, E., and Cox, W. M. (1987). Dimensions of thought flow in everyday life. Imagin. Cogn. Pers. 7, 125-128.

Mason, M. F., Norton, M. I., Van Horn, J. D., Wegner, D. M., Grafton, S. T., and Macrae, C. N. (2007). Wandering minds: the default network and stimulus-independent thought. Science 315, 393-395.

Matthews, G., Joyner, L., Gilliland, K. Campbell, S. E., Falconer, S., and Huggins, J. (1999). Validation of a comprehensive stress state questionnaire: towards a state "big three". Personal. Psychol. Eur. 7, 335-350.

Mazoyer, B., Zago, L., Mellet, E., Bricogne, S., Etard, O., Houde, O., Crivello, F. Joliot, M., Petit, L., and TzourioMazoyer, N. (2001). Cortical networks for working memory and executive functions sustain the conscious resting state in man. Brain Res. Bull. 54, 287-298.

Mcguire, P. K., Paulsau, E., Frackowiak, R. S., and Frith, C. D. (1996). Brain activity during stimulus independent thought. Neuroreport 7, 2095-2099.

McKiernan, K. A., D'angelo, B. R. Kaufman, J. N., and Binder, J. R. (2006). Interrupting the "stream of consciousness": an fMRI investigation. Neuroimage 29, 1185-1191.

Miles, L. K., Karpinska, K., Lumsden, J., and Macrae, C. N. (2010). The meandering mind: vection and mental time travel. PLoS ONE 5, e10825. doi: 10.1371/journal.pone.0010825

Morin, A. (2009). "Inner speech and consciousness," in The Encyclopedia of Consciousness, ed. W. Banks (NY: Elsevier), 389-402.

Nir, Y., Mukamel, R., Dinstein, I., Privman, E., Harel, M., Fisch, L., GelbardSagiv, H., Kipervasser, S., Andelman, F., and Neufeld, M. Y. (2008) Interhemispheric correlations of slow spontaneous neuronal fluctuations revealed in human sensory cortex. Nat. Neurosci. 11, 1100-1108.

Northoff, G., and Bermpohl, F. (2004). Cortical midline structures and the self. Trends Cogn. Sci. (Regul. Ed.) 8, 102.

Northoff, G., Heinzel, A., De Greck, M. Bermpohl, F., Dobrowolny, H., and Panksepp, J. (2006). Self-referential processing in our brain - a metaanalysis of imaging studies on the self. Neuroimage 31, 440-457.

Northoff, G., Qin, P., and Nakao, T. (2010). Rest-stimulus interaction in the brain: a review. Trends Neurosci.33,277-284.

Raichle, M.E. (2009). A paradigm shift in functional brain imaging. J. Neurosci. 29, 12729-12734.

Raichle, M. E., Macleod, A. M., Snyder, A. Z., Powers, W. J., Gusnard, D. A., and Shulman, G. L. (2001). A default mode of brain function. Proc. Natl. Acad. Sci. U.S.A. 98, 676-682.

Salvador, R., Suckling, J., Schwarzbauer, C., and Bullmore, E. (2005). Undirected graphs of frequency-dependent functional connectivity in whole brain networks. Philos. Trans. R. Soc. Lond. B Biol. Sci. 360, 937.

Schneider, F., Bermpohl, F., Heinzel, A., Rotte, M., Walter, M., Tempelmann, C., Wiebking, C., Dobrowolny, H., Heinze, H. J., and Northoff, G. (2008) The resting brain and our self: selfrelatedness modulates resting state neural activity in cortical midline structures. Neuroscience 157, 120-131.

Shmuel, A., and Leopold, D. A. (2008). Neuronal correlates of spontaneous fluctuations in fMRI signals in monkey visual cortex: implications for functional connectivity at rest. Hum. Brain Mapp. 29, 751-761.

Shmuel, A., Yacoub, E., Pfeuffer, J., Van De Moortele, P. F., Adriany, G., Hu, X., and Ugurbil, K. (2002). Sustained negative BOLD, blood flow and oxygen consumption response and its coupling to the positive response in the human brain. Neuron 36, 1195-1210.

Shulman, G. L., Corbetta, M., Buckner, R. L., Fiez, J.A., Miezin, F. M., Raichle, M. E., and Petersen, S. E. (1997). Common blood flow changes across visual tasks: I. Increases in subcortical structures and cerebellum but not in nonvisual cortex. J. Cogn. Neurosci. 9, 624-647.

Singer, J. L., and McCraven, V. G. (1961). Some characteristics of adult daydreaming. J. Psychol. 51, 151-164.

Smallwood, J., Brown, K. S., Tipper, C., Giesbrecht, B., Franklin, M.S., Mrazek, M. D., Carlson, J. M., and Schooler, J. W. (2011a).Pupillometric evidence for the decoupling of attention from perceptual input during offline thought. PLoS ONE 6, e18298. doi: 10.1371/ journal.pone.0018298

Smallwood, J., Schooler, J. W., Turk, D. J., Cunningham, S. J., Burns, P., and Macrae, C. N. (2011b). Self-reflection and the temporal focus of the wandering mind. Conscious. Cogn. (in press)

Smallwood, J., Mcspadden, M., and Schooler, J. W. (2008). When attention matters: the curious incident of the wandering mind. Mem. Cognit. $36,1144$.

Smallwood, J., Nind, L., and O'connor, R. C. (2009). When is your head at? An exploration of the factors associated with the temporal focus of the wandering mind. Conscious. Cogn. 18, 118-125.

Smallwood, J., and Schooler, J. W. (2006). The restless mind. Psychol. Bull. 132, 946.

Smallwood, J. M., Baracaia, S. F., Lowe, M. and Obonsawin, M. (2003). Task unre- 
lated thought whilst encoding information. Conscious. Cogn. 12, 452-484.

Spreng, R. N., Mar, R. A., and Kim, A. S. N. (2009). The common neural basis of autobiographical memory, prospection, navigation, theory of mind, and the default mode: a quantitative meta-analysis. J. Cogn. Neurosci. 21, 489-510.

Spreng, R.N., Stevens, W.D., Chamberlain, J. P., Gilmore, A. W., and Schacter, D. L. (2010). Default network activity, coupled with the frontoparietal control network, supports goal-directed cognition. Neuroimage 53, 303-317.

Stawarczyk, D., Majerus, S., Maquet, P., and D'Argembeau, A. (2011). Neural correlates of ongoing conscious experience: both task-unrelatedness and stimulus-independence are related to default network activity. PLoS ONE 6, e16997. doi: 10.1371/journal. pone.0016997

Stickgold, R., Hobson, J. A., Fosse, R., and Fosse, M. (2001). Sleep, learning, and dreams: off-line memory reprocessing. Science 294, 1052.

Teasdale, J. D., Dritschel, B. H., Taylor, M. J., Proctor, L., Lloyd, C. A., Nimmo-Smith, I., and Baddeley, A. D. (1995). Stimulus-independent thought depends on central executive resources. Mem. Cognit. 23, 551-551.

Tulving, E. (2005). Episodic Memory and Autonoesis: Uniquely Human? New York: Oxford University Press.

Uddin, L. Q., Clare Kelly, A., Biswal, B. B., Xavier Castellanos, F., and Milham, M. P. (2009). Functional connectivity of default mode network components: correlation, anticorrelation, and causality. Hum. Brain Mapp. 30, 625.

Van Den Heuvel, M., Mandl, R., and Pol, H. H. (2008). Normalized cut group clustering of resting-state FMRI data. PLoS ONE3, e2001.doi: 10.1371/journal.pone.0002001

Van Den Heuvel, M. P., and Hulshoff Pol, H. E. (2010). Exploring the brain network: a review on resting-state fMRI functional connectivity. Eur. Neuropsychopharmacol. 20, 519-534.

Wicker, B., Ruby, P., Royet, J.-P., and Fonlupt, P. (2003). A relation between rest and the self in the brain? Brain Res. Rev. 43, 224.

Conflict of Interest Statement: The authors declare that the research was conducted in the absence of any commercial or financial relationships that could be construed as a potential conflict of interest.

Received: 08 January 2011; accepted: 25 May 2011; published online: 06 June 2011.

Citation: Gruberger M, Ben-Simon E, Levkovitz $Y$, Zangen $A$ and Hendler $T$ (2011) Towards a neuroscience of mindwandering. Front. Hum. Neurosci. 5:56. doi: 10.3389/fnhum.2011.00056

Copyright (C) 2011 Gruberger, Ben-Simon, Levkovitz, Zangen and Hendler. This is an open-access article subject to a nonexclusive license between the authors and Frontiers Media SA, which permits use, distribution and reproduction in other forums, provided the original authors and source are credited and other Frontiers conditions are complied with. 


\section{APPENDIX}

Table A1 | A summary of overviewed studies and their non-DMN-related results.

Strategy/study
Brain region/BA

\begin{tabular}{|c|c|c|c|c|c|c|}
\hline \multicolumn{2}{|c|}{ Frontal cortex } & \multirow{2}{*}{$\begin{array}{l}\begin{array}{l}\text { Occipital } \\
\text { cortex }\end{array} \\
\text { Primary } \\
\text { visual areas }\end{array}$} & \multicolumn{3}{|c|}{ Temporal cortex } & \multirow{2}{*}{$\begin{array}{l}\text { Sub } \\
\text { cortica } \\
\text { Insula }\end{array}$} \\
\hline Motor areas & Broca's area & & $\begin{array}{l}\text { Temporal } \\
\text { pole }\end{array}$ & LTC & MTC & \\
\hline 6,4 & 44,45 & $17,18,19$ & 38 & $41,21,20$ & $35,36,29$ & \\
\hline $\mathrm{R}$ & L & & $R+L$ & $R+L$ & & $\mathrm{~L}$ \\
\hline \multirow[t]{3}{*}{ L } & & & & & $\mathrm{L}$ & \\
\hline & & Specific coor & nates not rep & & & \\
\hline & & & & & $R+L$ & \\
\hline \multirow[t]{3}{*}{$R+L$} & $R+L$ & & & & & $R+L$ \\
\hline & & & $R+L$ & $R+L$ & & \\
\hline & & & & & & $L$ \\
\hline \multirow[t]{2}{*}{ L } & & $R+L$ & L & & $R+L$ & $L$ \\
\hline & & $\mathrm{L}$ & & $\mathrm{L}$ & $\mathrm{L}$ & \\
\hline \multirow[t]{2}{*}{$\mathrm{R}$} & & L & & $R+L$ & $L$ & $R+L$ \\
\hline & & Specific coor & nates not rep & & & \\
\hline
\end{tabular}

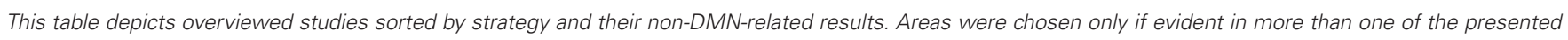
studies. Numbers represent Brodmann areas. LTC, lateral temporal cortex; MTC, medial temporal cortex.

A1. Real time MW sampling

Christoff et al. (2009), PNAS

Stawarczyk et al. (2011), Plos One

A2. Retrospective evaluation of MW

B1. Parametric modulation of self-relatedness

Gusnard et al. (2001), PNAS

Johnson et al. (2002), Brain

Goldberg et al. (2006), Neuron

Andrews-Hanna et al. (2010), Neuron

D'Argembeau et al. (2005), Neuroimage

Schneider et al. (2008), Neuroscience

B2. Parametric modulation of cognitive load

Wicker et al. (2003), Brain Res. Rev.

Christoff et al. (2004), Cortex

McKiernan et al. (2006), Neuroimage

Mason et al. (2007), Science

B3. Data analysis of neuronal dynamics 